\title{
Membrane and capillary components of lung diffusion and pro-angiogenic cells in infants
}

\author{
Daniel V. Chang ${ }^{1}$, Christina J. Tiller ${ }^{1}$, Jeffrey A. Kisling ${ }^{1}$, Jamie Case ${ }^{2,3,4,5}$, \\ Julie A. Mund ${ }^{2,3,4,5}$, Laura S. Haneline ${ }^{2,4,5}$, David A. Ingram $2,4,5,6$ \\ and Robert S. Tepper ${ }^{1,4,5}$

\begin{abstract}
Affiliations:
${ }^{1}$ Dept of Pediatric Pulmonology, Indiana University Medical Center, Indianapolis, IN, ${ }^{2}$ Dept of Pediatrics, Indiana University Medical Center, Indianapolis, IN,

${ }^{3}$ Indiana University Melvin and Bren Simon Cancer Center, Indiana University, Indianapolis, IN,

${ }^{4}$ James Whitcomb Riley Hospital for Children, Indianapolis, IN,

${ }^{5}$ Herman B. Wells Center for Pediatric Research, Indianapolis, IN, and

${ }^{6}$ Dept of Biochemistry and Molecular Biology, Indiana University School of Medicine, Indianapolis, IN, USA.
\end{abstract}

\section{Correspondence:}

R.S. Tepper, Section of Pulmonology, James Whitcomb Riley Hospital for Children, 702 Barnhill Drive, ROC 4270, Indianapolis, IN 46202-5225, USA.

E-mail: rtepperdiupui.edu

ABSTRACT Angiogenesis is a critical determinant of alveolarisation, which increases alveolar surface area and pulmonary capillary blood volume in infants; however, our understanding of this process is very limited. The purpose of our study was to measure the pulmonary membrane diffusion capacity (DM) and pulmonary capillary blood volume $(\mathrm{VC})$ components of the diffusing capacity of the lung for carbon monoxide (DLCO) in healthy infants and toddlers, and evaluate whether these components were associated with pro-angiogenic circulating haematopoietic stem/progenitor cells (pCHSPCs) early in life.

21 healthy subjects (11 males), 3-25 months of age, were evaluated. DLCO was measured under normoxic and hyperoxic conditions, and $D \mathrm{M}$ and $V \mathrm{C}$ were calculated. From $1 \mathrm{~mL}$ venous blood, pCHSPCs were quantified by multiparametric flow cytometry.

$D M$ and $V C$ increased with increasing body length; however, membrane resistance as a fraction of total resistance to pulmonary diffusion remained constant with somatic size. In addition, DLCO and VC, but not $D M$, increased with an increasing percentage of pCHSPCs.

The parallel increase in the membrane and vascular components of pulmonary diffusion is consistent with alveolarisation during this period of rapid lung growth. In addition, the relationship between pCHSPCs and VC suggest that pro-angiogenic cells may contribute to this vascular process.

@ERSpublications

Vascular components of pulmonary diffusion are associated with circulating pro-angiogenic cells in infants and toddlers http://ow.ly/r0k8A

For editorial comments see page 329.

Received: Jan 282013 | Accepted after revision: May 062013 | First published online: May 162013

Support statement: We acknowledge research support from The Gerber Foundation, Fremont, MI, USA (grant 99364).

Conflict of interest: Disclosures can be found alongside the online version of this article at www.erj.ersjournals.com

Copyright @ERS 2014 


\section{Introduction}

Gas exchange is the primary function of the lung. Therefore, lung growth and development early in life must increase the alveolar surface area and the pulmonary capillary blood volume $(V C)$ in order to meet the increasing requirements for exchange that occur with somatic growth. Early in life, lung volume is thought to increase primarily by the addition of alveoli, while in older children, lung volume increases primarily by the expansion of existing alveoli. We have previously demonstrated that the diffusing capacity of the lung for carbon monoxide ( $D \mathrm{LCO}$ ) and alveolar volume $(\mathrm{VA})$ increase with increasing body length in infants and toddlers; however, the ratio of DLCO/VA remained constant with lung growth early in life [1]. These physiological findings are consistent with lung growth occurring by the addition of alveoli, with comparable increases in alveolar surface area and VA. Pulmonary diffusion is determined by membrane and vascular components, which may not increase comparably during this period of rapid lung growth. The partitioning of the membrane and vascular components of diffusion has been assessed physiologically in older children and adults by measuring DLCO under conditions of normoxia and hyperoxia, as described initially by ROUGHTON and FORSTER [2]. These measurements have not previously been assessed in infants and toddlers.

Angiogenesis is a critical part of alveolar development. Treatment of neonatal animals with inhibitors of angiogenesis results in impaired alveolar development with reduced pulmonary capillary density and reduced radial alveolar counts [3]. Through paracrine stimulation of resident endothelial cells in vessel walls, angiogenic circulating haematopoietic stem/progenitor cells (CHSPCs) can stimulate angiogenesis in vascular injury models, promote alveolar development in murine models of lung disease, and are decreased in adults with vascular disease and chronic obstructive lung disease; and angiogenic cells have been proposed as a therapeutic target to stimulate lung growth in human lung disease [4-7]. We have previously demonstrated that the normal physiological increase in pro-angiogenic ( $p$ ) CHSPCs during pregnancy is impaired in females with gestational diabetes mellitus and pCHSPCs were lower in cord blood of mothers with gestational diabetes mellitus [8]. In addition, we have demonstrated that adolescents with diabetes mellitus have impaired endothelium-dependent vasodilation, which correlates with reductions in the ratio of pro-angiogenic to non-angiogenic circulating progenitor cells [9]. Evaluating angiogenic cells in infants has been limited by the previous need for large volumes of blood to identify angiogenic circulating progenitor cells; however, we have previously described a multiparameter flow cytometry technique to identify pCHSPCs in 1-mL blood samples [10,11]. The relationship of pCHSPCs and a physiological measurement of pulmonary vascular development has not previously been evaluated in infants.

The purpose of our study was to assess the membrane and vascular components of diffusion in healthy infants and toddlers, and to evaluate whether pulmonary diffusion early in life was associated with an increase in pCHSPCs. We hypothesised that if lung growth early in life occurs primarily by the addition of alveoli, then the membrane and capillary components would increase with increasing somatic size. However, the relative contributions of these two components would remain constant during this period of rapid lung growth. In addition, we hypothesised that an increased number of pCHSPCs would be associated with increased pulmonary vascular development, as assessed by DLCO and VC.

\section{Materials and methods}

\section{Subjects}

Subjects were recruited on the basis of advertisements in local publications in Indianapolis (IN, USA). All subjects were born at a gestational age $>37$ weeks, had no cardiorespiratory malformations and had a respiratory history negative for wheezing, asthma, treatment with asthma medications or hospitalisation for a respiratory illness. Subjects were evaluated at James Whitcomb Riley Hospital for Children (Indianapolis) while sleeping in a supine position with chloral hydrate sedation $\left(50-100 \mathrm{mg} \cdot \mathrm{kg}^{-1}\right)$. Oxygen saturation and heart rate were monitored during testing as recommended by the American Thoracic Society (ATS)/ European Respiratory Society (ERS) guidelines [12]. The study was approved by the institutional review board at Indiana University, Indianapolis, and informed parental consent was obtained. The recruitment period was between 2008 and 2012 .

\section{Measurements}

DLCO and VA were assessed using a 4-s induced respiratory pause at an inflation pressure of $30 \mathrm{cmH}_{2} \mathrm{O}$, previously described [1]. Subjects breathed through a facemask. Flow was measured by a pneumotachometer (Hans Rudolph, Shawnee, KS, USA) and gas concentrations were measured using a mass spectrometer (Perkin Elmer MGA-1100, Waltham, MA, USA). Measurements were obtained in replicate using a normoxic test gas $\left(0.3 \% \mathrm{C}^{18} \mathrm{O}, 4 \% \mathrm{SF}_{6}, 4 \% \mathrm{He}, 21 \% \mathrm{O}_{2}\right.$ and balance $\left.\mathrm{N}_{2}\right)$. Prior to obtaining measurements of DLCO using the hyperoxic test gas $\left(0.3 \% \mathrm{C}^{18} \mathrm{O}, 4 \% \mathrm{SF}_{6}, 4 \% \mathrm{He}\right.$ and balance $\left.\mathrm{O}_{2}\right)$, subjects breathed $90 \%$ oxygen for several breaths. An interval of $\geqslant 4$ min occurred between each measurement, as recommended by the ATS/ERS task force [13]. 
DLCO was calculated from the inspired volume of test gas and the alveolar concentration of carbon monoxide, which was the average concentration between $60 \%$ and $80 \%$ of the passive expired volume following the induced respiratory pause. VA was calculated by the dilution technique. The alveolar partial pressure of oxygen $\left(\mathrm{PAO}_{2}\right)$ was also obtained from the mass spectrometer. DLCO, $V \mathrm{~A}$ and $\mathrm{PAO}_{2}$ for high and low oxygen measurements were expressed as the mean of two or three measurements within $10 \%$. A blood sample was obtained for haemoglobin $(\mathrm{Hb})$ concentration ( $\mathrm{Hb} 201$; HemoCue, Lake Forest, CA, USA) and DLCO was adjusted for $\mathrm{Hb}$ as recommended by the ATS/ERS task force [13].

The pulmonary membrane diffusion capacity $(D M)$ and $V C$ were calculated from measurements of DLCO under high and low oxygen conditions, and the kinetic factor for the rate of carbon monoxide uptake by the red cells $(\theta)$ (equations $1-3)[2,14]$.

$$
\begin{gathered}
D \mathrm{M}=1 /\left(1 / D \mathrm{LCO}_{\mathrm{Low}}-1 / \theta \mathrm{CO} V \mathrm{C}\right) \\
V \mathrm{C}=\left(1 / \operatorname{CCO}_{\mathrm{High}}\right)-\left(1 / \theta \mathrm{CO}_{\mathrm{Low}}\right) /\left(1 / D \mathrm{LCO}_{\mathrm{High}}\right)-\left(1 / D \mathrm{LCO}_{\mathrm{Low}}\right) \\
1 / \theta \mathrm{CO}=0.0057 \times \mathrm{PAO}_{2}+0.75
\end{gathered}
$$

Pro-angiogenic circulating progenitor cells

CHSPCs were identified by the Indiana University Angiogenesis, Endothelial and Pro-Angiogenic Cell Core (Indiana University Melvin and Bren Simon Cancer Center, Indianapolis, IN, USA) using a seven-colour multiparametric flow cytometry method, as previously described [10,11]. From a 5-mL venous blood sample, mononuclear cells were isolated and stained with antibodies against cell surface antigens; CD34, $\mathrm{AC133,} \mathrm{CD} 31, \mathrm{CD} 45, \mathrm{CD} 16$ and CD14, as well as a viability marker, CD41a, and glycophorin A for the exclusion of dead cells, platelets and red blood cells. Fluorescence minus one controls were prepared as negative gating controls, and anti-mouse Ig CompBeads (BD Biosciences, Bedford, MA, USA) were stained with antibodies as single-colour compensation controls. pCHSPCs that co-express CD34, AC133, CD45 and $\mathrm{CD} 31\left(\mathrm{CD} 34^{\text {bright }} \mathrm{AC} 133^{+} \mathrm{CD} 45^{\mathrm{dim}} \mathrm{CD} 31^{+}\right.$) were analysed using a BD LSRII flow cytometer (BD Biosciences, San Jose, CA, USA) with a 405-nm violet laser, 488-nm blue laser and a 633-nm red laser. The data was exported as FCS 3.0 files and analysed using FlowJo software, version 9.5.3 (Tree Star, Ashland, OR, USA). AC133 negative non-angiogenic progenitors were also enumerated $\left(\mathrm{CD} 34^{\text {bright }} \mathrm{AC} 133^{-} \mathrm{CD} 45^{\mathrm{dim}} \mathrm{CD} 31^{+}\right)$to calculate a ratio of pro-angiogenic to non-angiogenic (n) CHSPCs. We have previously reported that an imbalance between these two populations, or a low pCHSPC to nCHSPC ratio, is associated with vascular dysfunction in patients with peripheral arterial disease $[10,11]$, as well as vascular dysfunction in mothers

\begin{tabular}{|c|c|c|c|c|}
\hline Subject & Sex & Age months & Body length $\mathrm{cm}$ & Race \\
\hline 1 & Male & 3.33 & 62.00 & Caucasian \\
\hline 2 & Female & 3.53 & 62.50 & Caucasian \\
\hline 3 & Male & 5.00 & 63.00 & Non-Caucasian \\
\hline 4 & Female & 6.23 & 64.50 & Non-Caucasian \\
\hline 5 & Male & 6.37 & 65.50 & Non-Caucasian \\
\hline 6 & Female & 6.43 & 62.00 & Caucasian \\
\hline 7 & Male & 6.57 & 66.00 & Non-Caucasian \\
\hline 8 & Male & 7.70 & 68.00 & Caucasian \\
\hline 9 & Male & 10.70 & 76.00 & Caucasian \\
\hline 10 & Female & 11.93 & 70.50 & Caucasian \\
\hline 11 & Female & 12.53 & 76.30 & Non-Caucasian \\
\hline 12 & Male & 13.23 & 77.50 & Caucasian \\
\hline 13 & Male & 14.47 & 74.57 & Non-Caucasian \\
\hline 14 & Female & 14.70 & 75.00 & Caucasian \\
\hline 15 & Female & 14.70 & 77.70 & Non-Caucasian \\
\hline 16 & Female & 14.70 & 78.30 & Non-Caucasian \\
\hline 17 & Male & 17.43 & 78.00 & Caucasian \\
\hline 18 & Female & 19.13 & 86.00 & Non-Caucasian \\
\hline 19 & Female & 25.57 & 84.50 & Caucasian \\
\hline 20 & Male & 27.80 & 87.00 & Caucasian \\
\hline 21 & Male & 28.10 & 86.00 & Caucasian \\
\hline
\end{tabular}
with gestational diabetes and adolescents with diabetes [8].

\section{TABLE 1 Demographics of subjects}









\section{Statistical analysis}

Statistical analysis was performed with SPSS Statistics 20 (SPSS, Chicago, IL, USA). Descriptive statistics included mean and range for continuous variables. The Shapiro-Wilk test was performed to determine normality. Log transformation was applied to non-normally distributed variables. The relationships between measured variables and body length, as well as adjustment for sex and race were evaluated and results expressed using multiple linear regressions. For all analyses, the level for statistical significance was set at 0.05 .

\section{Results}

We evaluated 21 healthy infants and toddlers (11 males and 10 females; 12 Caucasian and nine nonCaucasian). The group had a mean (range) age of 12.9 (3.3-28.1) months and there were no sex differences for age $(p=0.96)$, body length $(p=0.86)$ or race $(p=0.67)$. Demographics for the subjects are summarised in table 1 . The values of pulmonary diffusion obtained when breathing room air and high oxygen levels are summarised for each subject in table 2. For each of the subjects DLCO measured on high inspired oxygen was lower than DLCO measured on room air. The results for the multiple linear regression analysis with independent variables of body length, race, sex and pCHSPCs:nCHSPCs are summarised in table 3. DLCO increased with increasing body length. In addition, an increasing ratio of pCHSPCs:nCHSPCs was associated with increasing DLCO, while there were no significant associations between DLCO and sex or race. Both $D \mathrm{M}$ and $V \mathrm{C}$ also increased with increasing body length (fig. 1), while an increasing pCHSPC:nCHSPC ratio was associated with increasing $V \mathrm{C}$, but not $D \mathrm{M}$. In addition, $V \mathrm{C}$, but not $D \mathrm{M}$, was associated with sex $(\mathrm{p}=0.016)$ with males having a higher $V \mathrm{C}$ than females after adjusting for body length. Interestingly, the ratio of pCHSPCs:nCHSPCs was not associated with body length, sex, race or post-natal age $(\mathrm{p}=0.834$, $\mathrm{p}=0.945, \mathrm{p}=0.55$ and $\mathrm{p}=0.61$, respectively).

Membrane resistance $(R \mathrm{M})$ and capillary resistance $(R \mathrm{C})$ components were calculated as the reciprocals of the conductance for diffusion; individual values for $R \mathrm{M}$ and $R \mathrm{C}$ are listed in table 2. $R \mathrm{M}$ as a percentage of total resistance ( $R \mathrm{M} \%$ ) averaged $57 \%$ and was not associated with body length (fig. 2).

\section{Discussion}

This study is the first to measure both the membrane and capillary components of pulmonary diffusion capacity in healthy infants and toddlers. We found that DLCO and VC increased with increasing body length; however, the $R \mathrm{M}$ to diffusion expressed as a fraction of total pulmonary resistance to diffusion remained constant over the age range evaluated. In addition, this is the first study to demonstrate a relationship between pCHSPCs and a physiological assessment of parenchymal lung growth in infants and toddlers; a higher ratio of pCHSPC to nCHSPCs was associated with a higher DLCO, as well as a higher VC. These physiological findings suggest that there are parallel increases of the membrane and vascular components of pulmonary diffusion early in life, which is consistent with continued alveolarisation of the lung parenchyma during this period of rapid lung growth. Our results also suggest that pCHSPCs may be important in lung growth and development.

We are not aware of any previous measurements of $D M$ and $V C$ for infants and toddlers; therefore, we are not able to compare our results with other physiological studies. However, ZeltNer et al. [15] have published morphometric estimates of $V C$ in a limited number of subjects in this very young age group.

TABLE 3 Multiple linear regression results for pulmonary diffusion

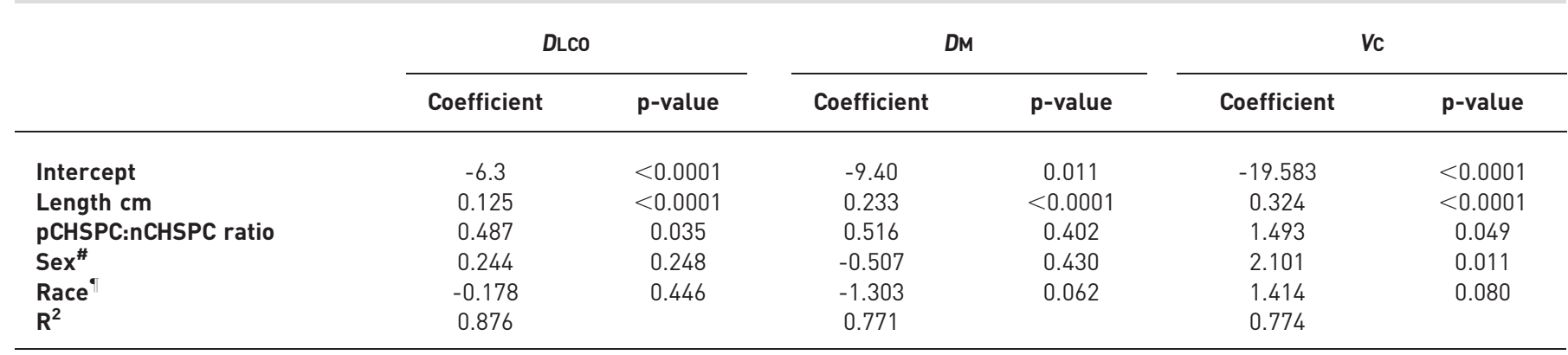

DLCO: diffusing capacity of the lung for carbon monoxide; DM: pulmonary membrane diffusion capacity; Vc: pulmonary capillary blood volume; pCHSPCs: pro-angiogenic circulating haematopoietic stem/progenitor cells; nCHSPCs: non-angiogenic circulating haematopoietic stem/progenitor cells. " : males were given a score of 1 and females a score of $0 ;{ }^{\circ}$ : non-Caucasians were given a score of 2 and Caucasians a score of 1 . 

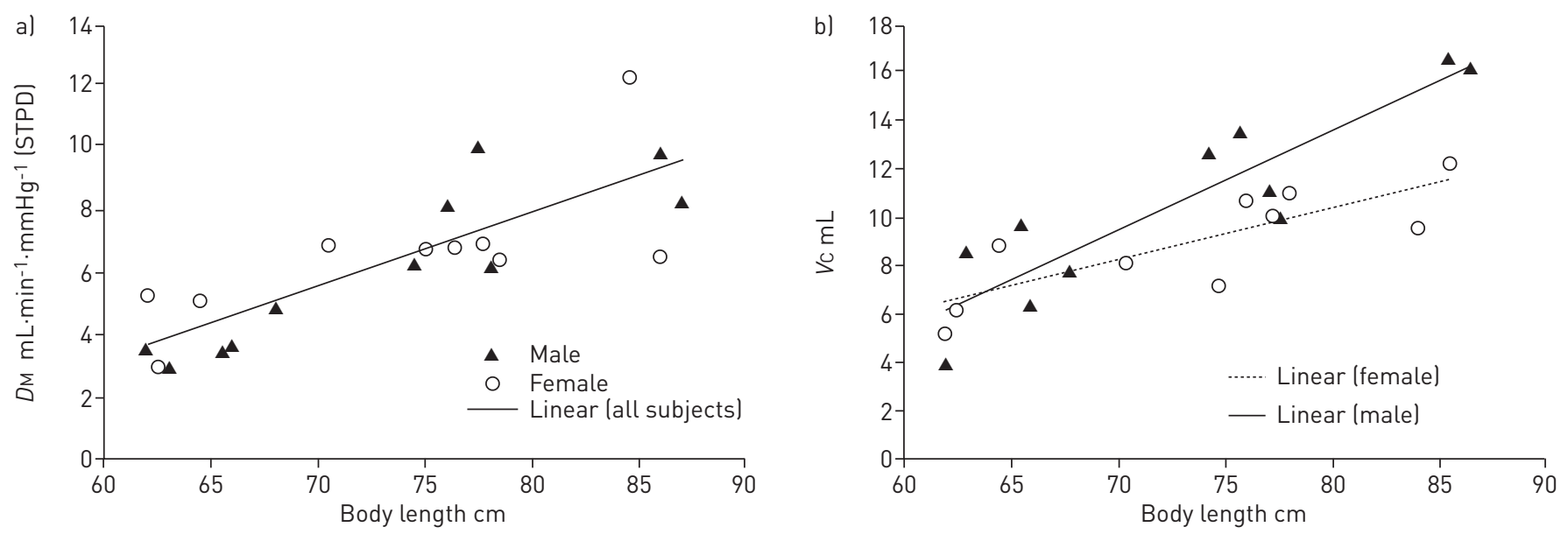

FIGURE 1 a) Pulmonary membrane diffusion capacity $(D M)$ versus body length for males $(n=11)$ and females $(n=10)$. There was a significant relationship between $D M$ and body length $\left(\mathrm{R}^{2} 0.673, \mathrm{p}<0.0001\right)$. STPD: standard temperature and pressure, dry (see table 2). b) Pulmonary capillary blood volume (VC) versus body length for males $(\mathrm{n}=11)$ and females $(\mathrm{n}=10)$. There was a significant relationship between $V$ C and body length $\left(\mathrm{R}^{2} 0.652, \mathrm{p}<0.0001\right)($ see table 2$)$.

For subjects with body lengths between $51 \mathrm{~cm}$ and $90 \mathrm{~cm}$, morphometric estimates of $V \mathrm{C}$ increased with increasing body length, similar to our data, and the morphometric estimates of $V \mathrm{C}$ ranged from $3 \mathrm{~mL}$ to $25 \mathrm{~mL}$. This morphometric data is comparable to our physiologic estimates of $V_{C}$ (fig. 1b), suggesting that our measurements of $\mathrm{VC}$ are reasonable.

We also found that DLCO, as well as the vascular component, but not the membrane component, increased with an increasing ratio of pCHSPC to nCHSPCs, after adjusting for somatic size, sex and race. We are not aware of any studies assessing the relationship between circulating angiogenic cells and lung growth in humans during this period of rapid lung growth early in life. In menstruating females, the dramatic vascular growth in the uterus is accompanied by an increase in circulating angiogenic cells and an associated increase in DLCO [16]. Studies using neonatal murine models of bronchopulmonary dysplasia (BPD), which exhibit impaired alveolar development, have demonstrated that infusion of angiogenic cells can restore alveolar development in this model, which suggests the potential importance of these cells in lung growth and development [6]. In addition, fewer pro-angiogenic circulating progenitor cells in cord blood of infants born very premature is associated with an increased risk of developing BPD, which is characterised by impaired alveolar development and decreased DLCO [17-19]. This finding of a relationship between circulating progenitor cells and vascular function in infants would be consistent with our previous report that having fewer CHSPCs was associated with decreased vascular function in adolescents with diabetes [9]. Mobilisation of circulating pro-angiogenic cells may be an effective strategy to induce lung growth, particularly in infants with impaired alveolar development; however, more mechanistic studies are required [4].

We did not find sex differences for DLCO, which is consistent with previous studies in this young age group that sex differences were not detected for DLCO, VA or functional residual capacity early in life when

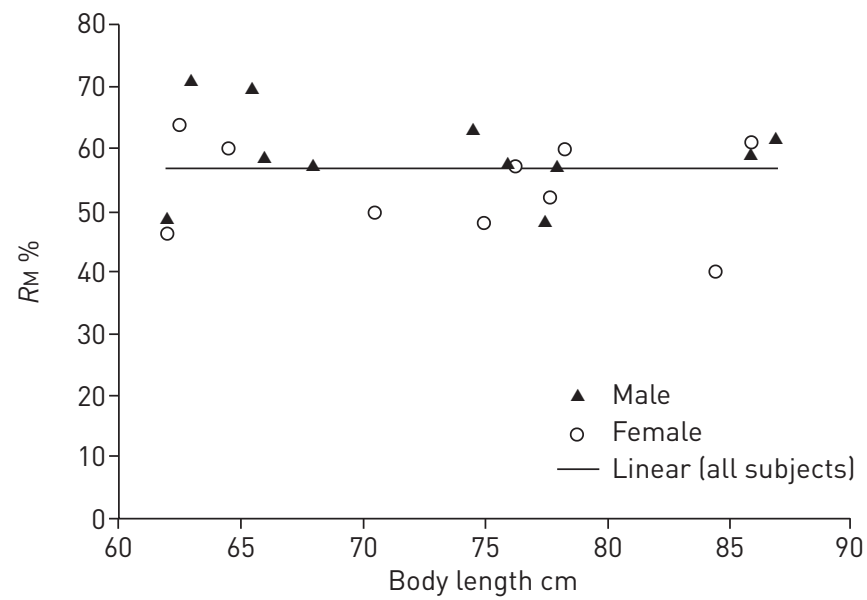

FIGURE 2 Membrane resistance $(R M)$ as a percentage of the total resistance versus body length for males $(\mathrm{n}=11)$ and females $(\mathrm{n}=10)$. There was no significant relationship between $R M$, expressed as a percentage of the total resistance, and body length $\left(R^{2} 0.027\right.$, $\mathrm{p}=0.474$ ). 
adjusting for somatic size $[1,20]$. We did find a sex difference for $V C$, but not for DM, after adjusting for body length and race. In adults, ZANEN et al. [14] reported that males had higher values for DM and VC compared to females, while BUCCI et al. [21] found no sex differences for either DM or VC when evaluating older children and adults. The inconsistencies among these studies for the effect of sex may be related to ZANEN et al. [14] evaluating a much larger number of subjects, which was limited only to adults.

There are several limitations to our study. Using a technique that we developed for this very young age group, there is a positive intrathoracic pressure during the induced respiratory pause manoeuvre, which could potentially alter the VC. However, as we found that $V \mathrm{C}$ increased with increasing somatic size and our physiological measurements of $V C$ were similar to morphometric data for subjects of comparable size, we believe that our physiological measurements provide a good estimate of $V C$ in this age group. Our results were obtained with sedated infants assessed in the supine position, and thus cannot readily be compared to data acquired from older children and adults, where measurements are obtained in the upright position. Gravitational differences in blood flow distribution could potentially contribute to differences in the relationships that we observed in our very young subjects and the reported data in older subjects. In addition, our data is cross-sectional; therefore, it does not actually reflect lung growth, which would require a longitudinal assessment of subjects. Lastly, the small number of subjects in our study limits our interpretation of potential relationships between pCHSPCs and demographic characteristics; future studies are needed to further evaluate the relationships between the vascular and membrane components of DLCO and pCHSPCs in infants and toddlers.

In summary, we describe the partitioning of DLCO into its DM and VC components for healthy infants and toddlers. We found that $D \mathrm{M}$ and $V \mathrm{C}$ increased with increasing body length. The $R \mathrm{M}$ as a fraction of the total resistance to pulmonary diffusion remained constant with somatic growth, which suggests parallel increases in the membrane and vascular components of pulmonary diffusion during this period of rapid lung growth and alveolarisation. In addition, DLCO and VC increased with an increasing ratio of pCHSPC to nCHSPCs, which connects the potential importance of pro-angiogenic cells with a physiological measurement that reflects pulmonary vascular development in this very young age. In contrast to morphometric measurements of autopsied lungs, these in vivo physiological measurements have the potential for longitudinal assessment of lung growth and development. Future studies are required to determine whether partitioning of the membrane and vascular components of pulmonary diffusion provide new insights into the pathophysiology of lung diseases associated with impaired alveolar development, such as chronic lung disease of infancy, as well as the potential of targeting pCHSPCs to stimulate lung growth.

\section{Acknowledgements}

We would like to thank Emily Sims of the Angiogenesis, Endothelial and Pro-Angiogenic Cell Core of the Indiana University Melvin and Bren Simon Cancer Center (Indianapolis, IN, USA) for processing the venous blood samples for this study.

\section{References}

1 Balinotti JE, Tiller CJ, Llapur CJ, et al. Growth of the lung parenchyma early in life. Am J Respir Crit Care Med 2009; 179: $134-137$.

2 Roughton FJ, Forster RE. Relative importance of diffusion and chemical reaction rates in determining rate of exchange of gases in the human lung, with special reference to true diffusing capacity of pulmonary membrane and volume of blood in the lung capillaries. J Appl Physiol 1957; 11: 290-302.

3 Jakkula M, Le Cras TD, Gebb S, et al. Inhibition of angiogenesis decreases alveolarization in the developing rat lung. Am J Physiol Lung Cell Mol Physiol 2000; 279: L600-L607.

4 Huertas A, Palange P. Circulating endothelial progenitor cells and chronic pulmonary diseases. Eur Respir J 2011; 37: 426-431.

5 Urbich C, Dimmeler S. Endothelial progenitor cells: characterization and role in vascular biology. Circ Res 2004; 95: 343-353.

6 Balasubramaniam V, Ryan SL, Seedorf GJ, et al. Bone marrow-derived angiogenic cells restore lung alveolar and vascular structure after neonatal hyperoxia in infant mice. Am J Physiol Lung Cell Mol Physiol 2010; 298: L315-L323.

Hill JM, Zalos G, Halcox JP, et al. Circulating endothelial progenitor cells, vascular function, and cardiovascular risk. N Engl J Med 2003; 348: 593-600.

8 Acosta JC, Haas DM, Saha CK, et al. Gestational diabetes mellitus alters maternal and neonatal circulating endothelial progenitor cell subsets. Am J Obstet Gynecol 2011; 204: 254.e8-254.e15.

9 DiMeglio LA, Tosh A, Saha C, et al. Endothelial abnormalities in adolescents with type 1 diabetes: a biomarker for vascular sequelae? J Pediatr 2010; 157: 540-546.

10 Estes ML, Mund JA, Ingram DA, et al. Identification of endothelial cells and progenitor cell subsets in human peripheral blood. Curr Protoc Cytom 2010; 9: 9.33.1-11.

11 Estes ML, Mund JA, Mead LE, et al. Application of polychromatic flow cytometry to identify novel subsets of circulating cells with angiogenic potential. Cytometry A 2010; 77: 831-839.

12 Gaultier C, Fletcher ME, Beardsmore C, et al. Respiratory function measurements in infants: measurement conditions. Working Group of the European Respiratory Society and the American Thoracic Society. Eur Respir J 1995; 8: 1057-1066. 
13 Macintyre N, Crapo RO, Viegi G, et al. Standardisation of the single-breath determination of carbon monoxide uptake in the lung. Eur Respir J 2005; 26: 720-735.

14 Zanen P, van der Lee L, van der Mark T, et al. Reference values for alveolar membrane diffusion capacity and pulmonary capillary blood volume. Eur Respir J 2001; 18: 764-769.

15 Zeltner TB, Caduff JH, Gehr P, et al. The postnatal development and growth of the human lung. I. Morphometry. Respir Physiol 1987; 67: 247-267.

16 Farha S, Asosingh K, Laskowski D, et al. Pulmonary gas transfer related to markers of angiogenesis during the menstrual cycle. J Appl Physiol 2007; 103: 1789-1795.

17 Maynard SE, Min JY, Merchan J, et al. Excess placental soluble fms-like tyrosine kinase 1 (sFlt1) may contribute to endothelial dysfunction, hypertension, and proteinuria in preeclampsia. J Clin Invest 2003; 111: 649-658.

18 Balinotti JE, Chakr VC, Tiller C, et al. Growth of lung parenchyma in infants and toddlers with chronic lung disease of infancy. Am J Respir Crit Care Med 2010; 181: 1093-1097.

19 Thébaud B, Abman SH. Bronchopulmonary dysplasia: where have all the vessels gone? Roles of angiogenic growth factors in chronic lung disease. Am J Respir Crit Care Med 2007; 175: 978-985.

20 Tepper RS, Reister T. Forced expiratory flows and lung volumes in normal infants. Pediatr Pulmonol 1993; 15 : 357-361.

21 Bucci G, Cook CD, Barrie H, et al. Studies of respiratory physiology in children. V. Total lung diffusion, diffusing capacity of pulmonary membrane, and pulmonary capillary blood volume in normal subjects from 7 to 40 years of age. J Pediatr 1961; 58: 820-828. 